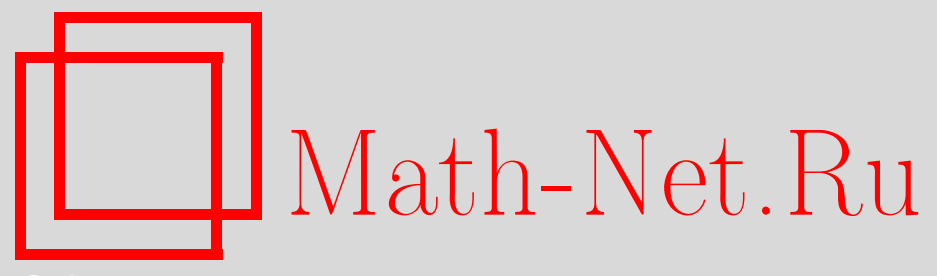

И. Г. Шапошников, Гомоморфные отношения многоосновных универсальных алгебр, Дискрет. матем., 2004, том 16, выпуск 4, 134-148

DOI: https://doi.org/10.4213/dm181

Использование Общероссийского математического портала Math-Net.Ru подразумевает, что вы прочитали и согласны с пользовательским соглашением http://www . mathnet.ru/rus/agreement

Параметры загрузки:

IP : 3.85 .5 .30

26 апреля 2023 г., 17:30:25

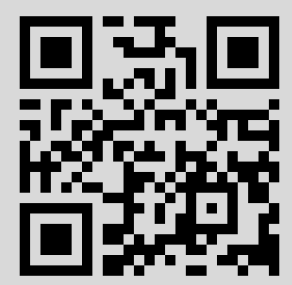


УдК 519.7

\title{
Гомоморфные отношения многоосновных универсальных алгебр
}

\author{
() 2004 г. И. Г. Шапошников
}

\begin{abstract}
Вводится понятие гомоморфного отношения многоосновных универсальных алгебр, для которого гомоморфизмы и $\pi$-гомоморфизмы являются частными случаями. Исследуются вопросы существования ряда новых отношений многоосновных универсальных алгебр.
\end{abstract}

\section{1. Введение}

В [1] было введено понятие гомоморфизма многоосновных универсальных алгебр (см. также [2]). Гомоморфизмы являются эффективным инструментом изучения алгебр. Вместе с тем нетривиальные гомоморфизмы существуют не у всех алгебр, или же имеющихся гомоморфизмов недостаточно для исследований. В этой связи в ряде работ были введены понятия, обобщающие понятия гомоморфизма. Например, в [3] было введено понятие $\pi$-гомоморфизма многоосновных универсальных алгебр, для которого при заданном распределении вероятностей на основных множествах гомоморфное соотношение выполняется с вероятностью, не меньшей $\pi$. В [4] было введено понятие скрещенного гомоморфизма, для которого определяются несколько гомоморфных соотношений, каждое из которых выполняется на своем подмножестве множества определения операций. Настоящая работа продолжает исследования в этом направлении. Содержанием статьи является исследование нового понятия гомоморфного отношения многоосновных универсальных алгебр, для которого гомоморфизмы и $\pi$-гомоморфизмы являются частными случаями. Везде далее мы будем рассматривать только конечные многоосновные универсальные алгебры.

С целью упрощения выкладок будем рассматривать многоосновные универсальные алгебры с одной операцией. Все полученные результаты понятным образом распространяются на произвольные многоосновные универсальные алгебры. Многоосновную универсальную алгебру с одной $n$-арной операцией

$$
\omega: A_{1} \times A_{2} \times \ldots \times A_{n} \rightarrow A_{0}
$$

мы будем обозначать $\left(A_{1}, A_{2}, \ldots, A_{n}, A_{0} ; \omega\right)$. Поскольку других алгебр, кроме многоосновных универсальных, у нас не появится, два этих прилагательных мы, как правило, будем опускать. Условимся под записью $\omega\left(A_{1}^{\prime}, A_{2}^{\prime}, \ldots, A_{n}^{\prime}\right)$, где $A_{i}^{\prime}$ - подмножество $A_{i}$,

$$
\omega: A_{1} \times A_{2} \times \ldots \times A_{n} \rightarrow A_{0}
$$


- некоторая операция, понимать множество $\left\{\omega\left(a_{1}, a_{2}, \ldots, a_{n}\right):\left(a_{1}, a_{2}, \ldots, a_{n}\right) \in A_{1}^{\prime} \times\right.$ $\left.A_{2}^{\prime} \times \ldots \times A_{n}^{\prime}\right\}$ элементов из $A_{0}$.

Следуя [5], отношение ядерной эквивалентности, связанное с отображением $\varphi$ множества $A$ в множество $B$, будем обозначать через $\operatorname{Ker} \varphi$, то есть $(x, y) \in \operatorname{Ker} \varphi$ тогда и только тогда, когда $\varphi(x)=\varphi(y), x, y \in A$. Класс эквивалентности множества $A$ с представителем $a \in A$ относительно отношения эквивалентности $\sigma$ будем обозначать $[a] \sigma$. Условимся говорить, что отношение эквивалентности $\sigma$ нетривиально, если разбиение на классы относительно $\sigma$ состоит из более чем одного класса.

Мы будем применять известные понятия алгебраической системы, модели и т.п., определенные в [6] для одноосновных алгебр, также и для многоосновных алгебр, не вводя новых определений там, где такое применение очевидно.

\section{2. Определение и свойства гомоморфных отношений многоосновных универсальных алгебр}

Определение 1. Предположим, что для $n$-арных операций

$$
\omega: A_{1} \times \ldots \times A_{n} \rightarrow A_{0}, \quad \vartheta: B_{1} \times \ldots \times B_{n} \rightarrow B_{0}, \quad\left|A_{i}\right| \geqslant\left|B_{i}\right|,
$$

определены отображения $\varphi_{i}$ множеств $A_{i}$ на $B_{i}$ и задано бинарное отношение $\rho$ на элементах множества $B_{0}^{N}$,

$$
N=\left|A_{1} \times \ldots \times A_{n}\right|
$$

Упорядочим некоторым образом элементы множества

$$
A_{1} \times \ldots \times A_{n}=\left\{\beta_{1}, \beta_{2}, \ldots, \beta_{N}\right\}
$$

и введем обозначения

$$
\gamma_{\vartheta \Phi}=\left(b_{1}, \ldots, b_{N}\right) \in B_{0}^{N}
$$

где

$$
b_{i}=\vartheta\left(\varphi_{1}\left(a_{1}\right), \ldots, \varphi_{n}\left(a_{n}\right)\right), \quad\left(a_{1}, \ldots, a_{n}\right)=\beta_{i}, \quad \gamma_{\Phi \omega}=\left(b_{1}^{\prime}, \ldots, b_{N}^{\prime}\right) \in B_{0}^{N}
$$

И

$$
b_{i}^{\prime}=\varphi_{0}\left(\omega\left(\beta_{i}\right)\right), \quad i=1, \ldots, N .
$$

Тогда, если $\gamma_{\vartheta} \Phi$ находится в отношении $\rho$ с $\gamma_{\Phi \omega}$, то будем говорить, что многоосновная универсальная алгебра $\left(A_{1}, \ldots, A_{n}, A_{0} ; \omega\right)$ находится в гомоморфном отношении $\rho$ с алгеброй $\left(B_{1}, \ldots, B_{n}, B_{0} ; \vartheta\right)$ при гомоморфизме $\Phi=\left(\varphi_{1}, \varphi_{2}, \ldots, \varphi_{n}, \varphi_{0}\right)$ отношения $\rho$.

Заметим, что в общем случае существование гомоморфного отношения $\rho$ может зависеть от способа упорядочения элементов множества $A_{1} \times \ldots \times A_{n}$. Поэтому, строго говоря, следует определять гомоморфное отношение $\rho$ при заданном упорядочении множества $A_{1} \times \ldots \times A_{n}$. Однако, так как в дальнейшем мы будем рассматривать только отношения $\rho$, для которых существование гомоморфных отношений многоосновных универсальных алгебр не зависит от упорядочения множества $A_{1} \times \ldots \times A_{n}$, данное уточнение 
в рассуждениях мы будем опускать. Такие гомоморфные отношения мы будем называть нормальными.

Выделим некоторые частные случаи нормальных гомоморфных отношений. Если в условиях определения 1 отношение $\rho$ на элементах множества $B_{0}^{N}$ индуцируется некоторым отношением $\rho_{0}$ на множестве $B_{0}$, то такое гомоморфное отношение будем называть координатным гомоморфным отношением $\rho_{0}$. В частности, если $\rho_{0}-$ отношение равенства, то это гомоморфное отношение есть просто гомоморфизм. Если $\rho_{0}-$ отношение неравенства, то такое координатное гомоморфное отношение будем называть инверсным гомоморфизмом. Пусть $\rho$ - отношение равенства точно $k$ (произвольных) координат элементов декартова произведения $B_{0}^{N}$, то есть на некотором собственном подмножестве $Q$ множества определения $A_{1} \times \ldots \times A_{n}$ мощности $k$ выполняется равенство

$$
\vartheta\left(\varphi_{1}\left(a_{1}\right), \ldots, \varphi_{n}\left(a_{n}\right)\right)=\varphi_{0}\left(\omega\left(a_{1}, \ldots, a_{n}\right)\right)
$$

Тогда такое гомоморфное отношение будем называть неточным гомоморфизмом, при этом величину

$$
p=\frac{k}{\left|A_{1} \times \ldots \times A_{n}\right|}
$$

будем называть значимостью неточного гомоморфизма.

Каждой координате векторов $\left\{\left(b_{1}, \ldots, b_{N}\right)\right\}$ множества $B_{0}^{N}$ взаимно однозначно поставим в соответствие $\zeta$ некоторое значение аргумента $\left(a_{1}, \ldots, a_{n}\right) \in A_{1} \times \ldots \times A_{n}$, то есть зададим отображение

$$
\zeta:\{1, \ldots, N\} \rightarrow A_{1} \times \ldots \times A_{n} .
$$

Пусть у неточного гомоморфизма множество координат $I$, на котором выполняется равенство, соответствует (при соответствии $\zeta$ ) объединению подмножеств вида $\alpha_{1} \times \ldots \times \alpha_{n}$, где $\alpha_{i}-$ класс эквивалентности относительно $\operatorname{Ker} \varphi_{i}$. Тогда такой неточный гомоморфизм будем называть ограниченным гомоморфизмом на ограничении $Q=\zeta(I)$. Заметим, что в данном случае отношение $\rho$ зависит от заданных отображений $Ф$. Данное отношение $\rho$ определяется следующим образом. Зафиксируем некоторое множество $Q$, являющееся некоторым объединением подмножеств вида $\alpha_{1} \times \ldots \times \alpha_{n}$, где $\alpha_{i}-$ класс эквивалентности относительно $\operatorname{Ker} \varphi_{i}$, и определим множество координат $I=\zeta^{-1}(Q)$. Отношение $\rho$ будет отношением равенства координат векторов из множества $I$.

Заметим, что $\pi$-гомоморфизм, введенный в [3], также является гомоморфизмом отношения $\rho$. Пусть на множестве определения $\omega$ задано некоторое распределение вероятностей и взаимно однозначное отображение

$$
\zeta:\{1, \ldots, N\} \rightarrow A_{1} \times \ldots \times A_{n} .
$$

Отношение $\rho$ есть отношение равенства векторов на координатах таких, что совокупная вероятность аргументов при соответствии $\zeta$, на которых оно выполняется, не менее $\pi$. Заметим, что в данном случае отношение $\rho$ зависит от заданного распределения вероятностей на $A_{1} \times \ldots \times A_{n}$. Так как $\pi$-гомоморфизмы не сводятся к гомоморфизмам алгебр и алгебраических систем, следовательно, гомомсрфные отношения не сводятся к гомоморфизмам алгебраических систем. Еще один пример, свидетельствующий об этом, будет приведен в разделе 3. 
Практическое использование введенных понятий аналогично использованию гомоморфизмов [7], $\pi$-гомоморфизмов [3] и скрещенных гомоморфизмов [4], например, для решения уравнений над алгебрами.

В дальнейшем элементы декартова произведения мы также будем называть векторами, что не должно вызвать недоразумений.

Спределение 2. Пусть

$$
\varepsilon(\omega)=\left(\varepsilon\left(A_{1}\right), \ldots, \varepsilon\left(A_{n}\right), \varepsilon\left(A_{0}\right)\right)
$$

- совокупность отношений эквивалентности на множествах $A_{1}, \ldots, A_{n}, A_{0}$ такая, что на векторах $\left(A_{0} / \varepsilon\left(A_{0}\right)\right)^{N}, N=\left|A_{1} \times \ldots \times A_{n}\right|$, определено отношение $\rho$ и для операции $\omega$ алгебры $\left(A_{1}, \ldots, A_{n}, A_{0} ; \omega\right)$ задано соответствие $\omega^{*}$ следующим образом: каждому набору классов $\left[a_{1}\right] \varepsilon\left(A_{1}\right), \ldots,\left[a_{n}\right] \varepsilon\left(A_{n}\right)$ поставлен в соответствие класс $\left[a_{0}\right] \varepsilon\left(A_{0}\right)$ так, что вектор $\left\{\omega\left(a_{i_{1}}, \ldots, a_{i_{n}}\right):\left(a_{i_{1}}, \ldots, a_{i_{n}}\right) \in A_{\mathrm{I}} \times \ldots \times A_{n}\right\}$ содержится в некотором векторе $\left\{\left[b_{i_{1}, \ldots, i_{n}}\right] \varepsilon\left(A_{0}\right)\right\}$, находящемся с вектором $\left\{\omega^{*}\left(\left[a_{i_{1}}\right] \varepsilon\left(A_{1}\right), \ldots,\left[a_{i_{n}}\right] \varepsilon\left(A_{n}\right)\right):\left(a_{i_{1}}, \ldots, a_{i_{n}}\right) \in\right.$ $\left.A_{1} \times \ldots \times A_{n}\right\}$ в отношении $\rho$.

Тогда пару $\left(\varepsilon(\omega), \omega^{*}\right)$ будем называть конгруэнцией отношения $\rho$ на алгебре $\left(A_{1}, \ldots, A_{n}, A_{0} ; \omega\right)$.

Заметим, что для совокупности отношений эквивалентности $\varepsilon(\omega)$ может, вообще говоря, существовать несколько различных конгруэнций отношения $\rho$, каждая из которых будет определяться своим соответствием $\omega^{*}$.

Для любой $\left(\varepsilon(\omega), \omega^{*}\right)$-конгруэнции отношения $\rho$ на алгебре $\left(A_{1}, \ldots, A_{n}, A_{0} ; \omega\right)$ можно определить фактор-алгебру $\left(A_{1} / \varepsilon\left(A_{1}\right), \ldots, A_{n} / \varepsilon\left(A_{n}\right), A_{0} / \varepsilon\left(A_{0}\right) ; \omega^{*}\right)$ отношения $\rho$ с операцией

$$
\omega^{*}: A_{1} / \varepsilon\left(A_{1}\right) \times \ldots \times A_{n} / \varepsilon\left(A_{n}\right) \rightarrow A_{0} / \varepsilon\left(A_{0}\right) .
$$

Из определения 2 следует, что совокупность канонических отображений

$$
\psi_{i}: A_{i} \rightarrow A_{i} / \varepsilon\left(A_{i}\right)
$$

любой конгруэнции $\left(\varepsilon(\omega), \omega^{*}\right)$ отношения $\rho$ есть гомоморфизм отношения $\rho$ алгебры $\left(A_{1}, \ldots, A_{n}, A_{0} ; \omega\right)$ на фактор-алгебру $\left(A_{1} / \varepsilon\left(A_{1}\right), \ldots, A_{n} / \varepsilon\left(A_{n}\right), A_{0} / \varepsilon\left(A_{0}\right) ; \omega^{*}\right)$ отношения $\rho$.

Теорема 1 (о гомоморфизме отношения $\rho$ ). Для отношений ядерных эквивалентностей

$$
\operatorname{Ker} \varphi=\left(\operatorname{Ker} \varphi_{1}, \ldots, \operatorname{Ker} \varphi_{n}, \operatorname{Ker} \varphi_{0}\right)
$$

любого гомоморфизма

$$
\varphi=\left(\varphi_{1}, \ldots, \varphi_{n}, \varphi_{0}\right)
$$

отношения $\rho$ алгебры $\left(A_{1}, \ldots, A_{n}, A_{0} ; \omega\right)$ на алгебру $\left(B_{1}, \ldots, B_{n}, B_{0} ; \vartheta\right)$ может быть задано такое соответствие

$$
\omega^{*}:\left(\left[a_{1}\right] \operatorname{Ker} \varphi_{1}, \ldots,\left[a_{n}\right] \operatorname{Ker} \varphi_{n}\right) \rightarrow\left[a_{0}\right] \operatorname{Ker} \varphi_{0},
$$

что $\left(\operatorname{Ker}(\varphi), \omega^{*}\right)$ есть конгруэнчия отношения $\rho^{*}$ на алгебру $\left(A_{1}, \ldots, A_{n}, A_{0} ; \omega\right)$ такого, что модели $\left(\left(B_{0}\right)^{N} ; \rho\right)$ u $\left(\left(A_{0} / \operatorname{Ker} \varphi_{0}\right)^{N} ; \rho^{*}\right), N=\left|A_{1} \times \ldots \times A_{n}\right|$, изоморфны. 
Совокупность канонических отображений

$$
\psi_{i}: A_{i} \rightarrow A_{i} / \varepsilon\left(A_{i}\right), \quad i=0,1, \ldots, n
$$

есть гомоморфизм отночения $\rho^{*}$ алгебры $\left(A_{1}, \ldots, A_{n}, A_{0} ; \omega\right)$ на фактор-алгебру $\left(A_{1} / \operatorname{Ker} \varphi_{1}, \ldots, A_{n} / \operatorname{Ker} \varphi_{n}, A_{0} / \operatorname{Ker} \varphi_{0} ; \omega^{*}\right)$ отношения $\rho^{*} ; n р и$ этом алгебраические системы $\left(A_{1} / \operatorname{Ker} \varphi_{1}, \ldots, A_{n} / \operatorname{Ker} \varphi_{n}, A_{0} / \operatorname{Ker} \varphi_{0},\left(A_{0} / \operatorname{Ker} \varphi_{0}\right)^{N} ; \omega^{*} ; \rho^{*}\right) u\left(B_{1}, \ldots, B_{n}, B_{0}\right.$, $\left.\left(B_{0}\right)^{N} ; \vartheta ; \rho\right)$ изоморфны.

Доказательство. На векторах $\left(A_{0} / \operatorname{Ker} A_{0}\right)^{N}, N=A_{1} \times \ldots \times A_{n}$, определим отношение $\rho^{*}$, полагая, что $\left(\left[a_{i_{1}}\right] \operatorname{Ker} \varphi_{0}, \ldots,\left[a_{i_{N}}\right] \operatorname{Ker} \varphi_{0}\right),\left(\left[a_{j_{1}}\right] \operatorname{Ker} \varphi_{0}, \ldots,\left[a_{j_{N}}\right] \operatorname{Ker} \varphi_{0}\right) \in \rho^{*}$ тогда и только тогда, когда $\left(\left(\varphi_{0}\left(a_{i_{1}}\right), \ldots, \varphi_{0}\left(a_{i_{N}}\right)\right),\left(\varphi_{0}\left(a_{j_{1}}\right), \ldots, \varphi_{0}\left(a_{j_{N}}\right)\right) \in \rho\right.$.

Нетрудно видеть, что отображение

$$
\tau_{0}: A_{0} / \sigma\left(\varphi_{0}\right) \rightarrow B_{0}
$$

ставящие в соответствие каждому классу $\left[a^{\prime}\right] \sigma\left(\varphi_{0}\right)$ элемент $b=\varphi_{0}\left(a^{\prime}\right)$, есть изоморфизм моделей $\left(\left(B_{0}\right)^{N} ; \rho\right)$ и $\left(\left(A_{0} / \operatorname{Ker} \varphi_{0}\right)^{N} ; \rho^{*}\right)$.

Каждому набору $\left[a_{1}\right] \operatorname{Ker} \varphi_{1}, \ldots,\left[a_{n}\right] \operatorname{Ker} \varphi_{n}$ поставим в соответствие $\omega^{*}$ класс $\left[a_{0}\right] \operatorname{Ker} \varphi_{0}$, для которого

$$
\vartheta\left(\varphi_{1}\left(a_{1}\right), \ldots, \varphi_{n}\left(a_{n}\right)\right)=\varphi_{0}\left(a_{0}\right) .
$$

Тогда из условия, что векторы $\left\{\vartheta\left(\varphi_{1}\left(a_{1}\right), \ldots, \varphi_{n}\left(a_{n}\right)\right):\left(a_{1}, \ldots, a_{n}\right) \in A_{1} \times \ldots \times A_{n}\right\}$, $\left\{\varphi_{0}\left(\omega\left(a_{1}, \ldots, a_{n}\right)\right):\left(a_{1}, \ldots, a_{n}\right) \in A_{1} \times \ldots \times A_{n}\right\}$ находятся в отношении $\rho$ (условия гомоморфизма отношения $\rho)$ следует, что вектор $\left\{\omega\left(a_{i_{1}}, \ldots, a_{i_{n}}\right):\left(a_{1}, \ldots, a_{n}\right) \in A_{1} \times \ldots \times A_{n}\right\}$ содержится в некотором векторе $\left\{\left[b_{i_{1}, \ldots, i_{n}}\right] \operatorname{Ker} \varphi_{0}\right\}$, находящимся в отношении $\rho^{*}$ с вектором $\left\{\omega^{*}\left(\left[a_{i_{1}}\right] \operatorname{Ker} \varphi_{1}, \ldots,\left[a_{i_{n}}\right] \operatorname{Ker} \varphi_{n}\right):\left(a_{1}, \ldots, a_{n}\right) \in A_{1} \times \ldots \times A_{n}\right\}$. Следовательно, $\left(\operatorname{Ker}(\varphi), \omega^{*}\right)-$ конгруэнция отношения $\rho^{*}$ на $\left(A_{1}, \ldots, A_{n}, A_{0} ; \omega\right)$, и определена факторалгебра $\left(A_{1} / \operatorname{Ker} \varphi_{1}, \ldots, A_{n} / \operatorname{Ker} \varphi_{n}, A_{0} / \operatorname{Ker} \varphi_{0} ; \omega^{*}\right)$ отношения $\rho^{*}$.

Из вышеприведенных рассуждений следует, что совокупность канонических отображений

$$
\psi_{i}: A_{i} \rightarrow A_{i} / \operatorname{Ker} \varphi_{i}, \quad i=0,1, \ldots, n,
$$

есть гомоморфизм отношения $\rho^{*}$ алгебры $\left(A_{1}, \ldots, A_{n}, A_{0} ; \omega\right)$ на фактор-алгебру $\left(A_{1} / \operatorname{Ker} \varphi_{1}, \ldots, A_{n} / \operatorname{Ker} \varphi_{n}, A_{0} / \operatorname{Ker} \varphi_{0} ; \omega^{*}\right)$ отношения $\rho^{*}$. Из соотношения (1) следует, что отображения

$$
\tau_{i}: A_{i} / \operatorname{Ker} \varphi_{i} \rightarrow B_{i}
$$

ставящие в соответствие каждому классу $\left[a_{i}\right] \operatorname{Ker} \varphi_{i}$ элемент $b=\varphi_{i}\left(a_{i}\right)$ есть изоморфизм алгебраических систем $\left(A_{1} / \operatorname{Ker} \varphi_{1}, \ldots, A_{n} / \operatorname{Ker} \varphi_{n}, A_{0} / \operatorname{Ker} \varphi_{0},\left(A_{0} / \operatorname{Ker} \varphi_{0}\right)^{N} ; \omega^{*} ; \rho^{*}\right)$ и $\left(B_{1}, \ldots, B_{n}, B_{0},\left(B_{0}\right)^{N} ; \vartheta ; \rho\right)$. Теорема доказана.

Заметим, что теорема 1 уточняет теорему о $\pi$-гомоморфизмах работы [3]. Из теоремы 1 следует, что задача описания с точностью до изоморфизма гомоморфных образов отношения $\rho$ алгебры $\left(A_{1}, \ldots, A_{n}, A_{0} ; \omega\right)$ эквивалентна описанию всех ее конгруэнций отношения $\rho$. В этой связи приведем определения конгруэнций отношений для выше выделенных частных случаев в более удобном для использования виде. Эквивалентность ниже приводимых определений определению 2 легко устанавливается. 
Отношения эквивалентности

$$
\varepsilon(\omega)=\left(\varepsilon\left(A_{1}\right), \ldots, \varepsilon\left(A_{n}\right), \varepsilon\left(A_{0}\right)\right)
$$

на множествах $A_{1}, \ldots, A_{n}, A_{0}$ есть конгруэнция алгебры $\left(A_{1}, \ldots, A_{n}, A_{0} ; \omega\right)$, если для любых классов $\left[a_{1}\right] \varepsilon\left(A_{1}\right), \ldots,\left[a_{n}\right] \varepsilon\left(A_{n}\right)$ найдется класс $\left[a_{0}\right] \varepsilon\left(A_{0}\right)$ такой, что (см. [7])

$$
\omega\left(\left[a_{1}\right] \varepsilon\left(A_{1}\right), \ldots,\left[a_{n}\right] \varepsilon\left(A_{n}\right)\right) \subset\left[a_{0}\right] \varepsilon\left(A_{0}\right) .
$$

Пусть

$$
\varepsilon(\omega)=\left(\varepsilon\left(A_{1}\right), \ldots, \varepsilon\left(A_{n}\right), \varepsilon\left(A_{0}\right)\right)
$$

- отношения эквивалентности на основных множествах $A_{1}, \ldots, A_{n}, A_{0}$ алгебры $\left(A_{1}, \ldots, A_{n}, A_{0} ; \omega\right)$ и задано соответствие $\omega^{*}$ следующим образом: каждому набору классов вида $\left[a_{1}\right] \varepsilon\left(A_{1}\right), \ldots,\left[a_{n}\right] \varepsilon\left(A_{n}\right)$ поставлен в соответствие класс $\left[a_{0}\right] \varepsilon\left(A_{0}\right)$ так, что выполняется условие

$$
\sum_{\left(a_{1}, \ldots, a_{n}\right)}\left|\left\{\left(b_{1}, \ldots, b_{n}\right): b_{i} \in\left[a_{i}\right] \varepsilon\left(A_{i}\right), i=1, \ldots, n, \omega\left(b_{1}, \ldots, b_{n}\right) \in\left[a_{0}\right] \varepsilon\left(A_{0}\right)\right\}\right|=p,
$$

где суммирование проводится по всем представителям классов эквивалентности $\varepsilon\left(A_{1}\right), \ldots, \varepsilon\left(A_{n}\right)$. Тогда пара $\left(\varepsilon(\omega), \omega^{*}\right)$ будет неточной конгруэнцией алгебры $\left(A_{1}, \ldots, A_{n}, A_{0} ; \omega\right)$ значимости $p$.

Пусть на множестве $A_{1} \times \ldots \times A_{n}$ задано распределение вероятностей $\mathbf{P}$,

$$
\varepsilon(\omega)=\left(\varepsilon\left(A_{1}\right), \ldots, \varepsilon\left(A_{n}\right), \varepsilon\left(A_{0}\right)\right)
$$

- отношения эквивалентности на основных множествах $A_{1}, \ldots, A_{n}, A_{0}$ алгебры $\left(A_{1}, \ldots, A_{n}, A_{0} ; \omega\right)$ и задано соответствие $\omega^{*}$ следующим образом: каждому набору классов вида $\left[a_{1}\right] \varepsilon\left(A_{1}\right), \ldots,\left[a_{n}\right] \varepsilon\left(A_{n}\right)$ поставлен в соответствие класс $\left[a_{0}\right] \varepsilon\left(A_{0}\right)$ так, что выполняется условие

$$
\sum_{\left(a_{1}, \ldots, a_{n}\right)} \mathbf{P}\left\{\left(b_{1}, \ldots, b_{n}\right): b_{i} \in\left[a_{i}\right] \varepsilon\left(A_{i}\right), i=1, \ldots, n, \omega\left(b_{1}, \ldots, b_{n}\right) \in\left[a_{0}\right] \varepsilon\left(A_{0}\right)\right\} \geqslant \pi,
$$

где суммирование проводится по всем представителям классов эквивалентности $\varepsilon\left(A_{1}\right), \ldots, \varepsilon\left(A_{n}\right)$. Тогда пара $\left(\varepsilon(\omega), \omega^{*}\right)-\pi$-конгруэнция алгебры $\left(A_{1}, \ldots, A_{n}, A_{0} ; \omega\right)$. Заметим, что данное определение эквивалентно определению, введенному в [3]. Отличие заключается в том, что в определении [3] явно не указывается соответствие $\omega^{*}$, хотя его сушествование подразумевается, так как в противном случае $\pi$-конгруэнция определяется неоднозначно.

Пусть

$$
\varepsilon(\omega)=\left(\varepsilon\left(A_{1}\right), \ldots, \varepsilon\left(A_{n}\right), \varepsilon\left(A_{0}\right)\right)
$$

- совокупность отношений эквивалентности на множествах $A_{1}, \ldots, A_{n}, A_{0}$ такая, что на фактор-множестве $A_{0} / \varepsilon\left(A_{0}\right)$ определено отношение $\rho$ и для операции $\omega$ алгебры $\left(A_{1}, \ldots, A_{n}, A_{0} ; \omega\right)$ задано соответствие $\omega^{*}$ следующим образом: каждому набору классов $\left[a_{1}\right] \varepsilon\left(A_{1}\right), \ldots,\left[a_{n}\right] \varepsilon\left(A_{n}\right)$ поставлен в соответствие класс $\left[a_{0}\right] \varepsilon\left(A_{0}\right)$ такой, что

$$
\omega\left(\left[a_{1}\right] \varepsilon\left(A_{1}\right), \ldots,\left[a_{n}\right] \varepsilon\left(A_{n}\right)\right) \subset \bigcup_{h}[b] \varepsilon\left(A_{0}\right),
$$


где объединение проводится по всем представителям классов $b \in A_{0}$ таким, что $\left([b] \varepsilon\left(A_{0}\right),\left[a_{0}\right] \varepsilon\left(A_{0}\right)\right) \in \rho$. Тогда пара $\left(\varepsilon(\omega), \omega^{*}\right)$ будет конгруэнцией координатного отношения $\rho$ на алгебре $\left(A_{1}, \ldots, A_{n}, A_{0} ; \omega\right)$.

Отношения эквивалентности

$$
\varepsilon(\omega)=\left(\varepsilon\left(A_{1}\right), \ldots, \varepsilon\left(A_{n}\right), \varepsilon\left(A_{0}\right)\right)
$$

на множествах $A_{1}, \ldots, A_{n}, A_{0}$ есть инверсная конгруэнция алгебры $\left(A_{1}, \ldots, A_{n}, A_{0} ; \omega\right)$, если для любых классов $\left[a_{1}\right] \varepsilon\left(A_{1}\right), \ldots,\left[a_{n}\right] \varepsilon\left(A_{n}\right)$ найдется класс $\left[a_{0}\right] \varepsilon\left(A_{0}\right)$ такой, что

$$
\omega\left(\left[a_{1}\right] \varepsilon\left(A_{1}\right), \ldots,\left[a_{n}\right] \varepsilon\left(A_{n}\right)\right) \subset A_{0} \backslash\left[a_{0}\right] \varepsilon\left(A_{0}\right) .
$$

Пусть

$$
\varepsilon(\omega)=\left(\varepsilon\left(A_{1}\right), \ldots, \varepsilon\left(A_{n}\right), \varepsilon\left(A_{0}\right)\right)
$$

- отношения эквивалентности на основных множествах $A_{1}, \ldots, A_{n}, A_{0}$ алгебры $\left(A_{1}, \ldots, A_{n}, A_{0} ; \omega\right)$ и для наборов классов $\left[a_{i_{1}}\right] \varepsilon\left(A_{1}\right), \ldots,\left[a_{i_{n}}\right] \varepsilon\left(A_{n}\right),\left(i_{1}, \ldots, i_{n}\right) \in Y$, найдется класс $\left[a_{i_{0}}\right] \varepsilon\left(A_{0}\right)$ такой, что $\omega\left(\left[a_{1}\right] \varepsilon\left(A_{1}\right), \ldots,\left[a_{n}\right] \varepsilon\left(A_{n}\right)\right) \subset\left[a_{0}\right] \varepsilon\left(A_{0}\right)$. Тогда $\varepsilon(\omega)$ - ограниченная конгруэнция алгебры $\left(A_{1}, \ldots, A_{n}, A_{0} ; \omega\right)$ на ограничении

$$
Q=\bigcup_{\left(i_{1}, \ldots, i_{n}\right) \in Y}\left[a_{i_{1}}\right] \varepsilon\left(A_{1}\right) \times \ldots \times\left[a_{i_{n}}\right] \varepsilon\left(A_{n}\right) .
$$

В [7] было введено понятие максимальной конгруэнции. Максимальные конгруэнции зачастую проще описывать (см., например, [4], [7]), а описания их достаточно для описания всех остальных конгруэнций. Аналогичное понятие можно ввести и для некоторых конгруэнций отношений. Например, из определения $\pi$-конгруэнции следует, что если $\varepsilon(\omega)=\left(\varepsilon\left(A_{1}\right), \ldots, \varepsilon\left(A_{n}\right), \varepsilon\left(A_{0}\right)\right)-\pi$-конгруэнция на алгебре $\left(A_{1}, A_{2}, \ldots, A_{n}, A_{0} ; \omega\right)$ и $\mu_{1}, \ldots, \mu_{n}, \mu_{0}$ - такие отношения эквивалентности на множествах $A_{1}, \ldots, A_{n}, A_{0}$, соответственно, что $\mu_{1} \subset \varepsilon\left(A_{1}\right), \ldots, \mu_{n} \subset \varepsilon\left(A_{n}\right), \mu_{0} \subset \varepsilon\left(A_{0}\right)$, то $\left(\mu_{1}, \ldots, \mu_{n}, \mu_{0}\right)$ также является $\pi$-конгруэнцией на алгебре $\left(A_{1}, A_{2}, \ldots, A_{n}, A_{0} ; \omega\right)$. В этом случае будем говорить, что $\pi$-конгруэнция $\left(\mu_{1}, \ldots, \mu_{n}, \mu_{0}\right)$ является следствием конгруэнции $\varepsilon(\omega)$. На множестве $\pi$-конгруэнций $\pi$-Con $(\omega)$ на алгебре $\left(A_{1}, A_{2}, \ldots, A_{n}, A_{0} ; \omega\right)$ введем отношение $\leqslant$ следующего вида:

$$
\left(\mu\left(A_{1}\right), \ldots, \mu\left(A_{n}\right), \mu\left(A_{0}\right)\right) \leqslant\left(\varepsilon\left(A_{1}\right), \ldots, \varepsilon\left(A_{n}\right), \varepsilon\left(A_{0}\right)\right)
$$

если

$$
\mu\left(A_{1}\right) \subset \varepsilon\left(A_{1}\right), \ldots, \mu\left(A_{n}\right) \subset \varepsilon\left(A_{n}\right), \quad \varepsilon\left(A_{0}\right) \subset \mu\left(A_{0}\right) .
$$

Нетрудно заметить, что $\leqslant-$ отношение частичного порядка. Действительно, из определения отношения $\leqslant$ очевидно следует его рефлексивность и антисимметричность. Покажем транзитивность. Пусть

$$
\begin{aligned}
& \left(\varepsilon_{1}\left(A_{1}\right), \ldots, \varepsilon_{1}\left(A_{n}\right), \varepsilon_{1}\left(A_{0}\right)\right) \leqslant\left(\varepsilon_{2}\left(A_{1}\right), \ldots, \varepsilon_{2}\left(A_{n}\right), \varepsilon_{2}\left(A_{0}\right)\right), \\
& \left(\varepsilon_{2}\left(A_{1}\right), \ldots, \varepsilon_{2}\left(A_{n}\right), \varepsilon_{2}\left(A_{0}\right)\right) \leqslant\left(\varepsilon_{3}\left(A_{1}\right), \ldots, \varepsilon_{3}\left(A_{n}\right), \varepsilon_{3}\left(A_{0}\right)\right) .
\end{aligned}
$$

Следовательно,

$$
\begin{aligned}
\varepsilon_{1}\left(A_{1}\right) \subset \varepsilon_{2}\left(A_{1}\right) \subset \varepsilon_{3}\left(A_{1}\right), \\
\ldots \\
\varepsilon_{1}\left(A_{n}\right) \subset \varepsilon_{2}\left(A_{n}\right) \subset \varepsilon_{3}\left(A_{n}\right), \\
\varepsilon_{3}\left(A_{0}\right) \subset \varepsilon_{2}\left(A_{0}\right) \subset \varepsilon_{1}\left(A_{0}\right),
\end{aligned}
$$


то есть

$$
\left(\varepsilon_{1}\left(A_{1}\right), \ldots, \varepsilon_{1}\left(A_{n}\right), \varepsilon_{1}\left(A_{0}\right)\right) \leqslant\left(\varepsilon_{3}\left(A_{1}\right), \ldots, \varepsilon_{3}\left(A_{n}\right), \varepsilon_{3}\left(A_{0}\right)\right) .
$$

Из определения отношения $\leqslant$ следует, что совокупность всех $\pi$-конгруэнций на алгебpe $\left(A_{1}, A_{2}, \ldots, A_{n}, A_{0} ; \omega\right)$, не являющихся следствием друг друга, исчерпывается максимальными элементами относительно $\leqslant$. Такие $\pi$-конгруэнции будем называть максимальными. Заметим, что любые две $\pi$-конгруэнции $\left(\mu_{1}, \ldots, \mu_{n}, \mu_{0}\right),\left(\varepsilon\left(A_{1}\right), \ldots, \varepsilon\left(A_{n}\right), \varepsilon\left(A_{0}\right)\right)$ на алгебре $\left(A_{1}, A_{2}, \ldots, A_{n}, A_{0} ; \omega\right)$ имеют нижнюю границу относительно $\leqslant$, а именно, $\pi$-конгруэнцию $\left(\mu\left(A_{1}\right) \cap \varepsilon\left(A_{1}\right), \ldots, \mu\left(A_{n}\right) \cap \varepsilon\left(A_{n}\right), \mu\left(A_{0}\right) \cup \varepsilon\left(A_{0}\right)\right)$. Следовательно, множество $\pi$-Con $(\omega)$ является нижней полурешеткой относительно $\leqslant$. В общем случае $\pi$-Con $(\omega)$ не является решеткой относительно $\leqslant$. Действительно, пусть на алгебре $\left(A_{1}, A_{2}, \ldots, A_{n}, A_{0} ; \omega\right)$ существуют две различные максимальные $\pi$-конгруэнции, они являются максимальными элементами $\pi-\operatorname{Con}(\omega)$, поэтому для них не существует верхней грани, а множество $\pi$-Con $(\omega)$ не является решеткой относительно $\leqslant$.

Заметим, что если

$$
\varepsilon(\omega)=\left(\varepsilon\left(A_{1}\right), \ldots, \varepsilon\left(A_{n}\right), \varepsilon\left(A_{0}\right)\right)
$$

- инверсная конгруэнция на алгебре $\left(A_{1}, A_{2}, \ldots, A_{n}, A_{0} ; \omega\right)$ и $\mu_{1}, \ldots, \mu_{n}, \mu_{0}$ - такие отношения эквивалентности на множествах $A_{1}, \ldots, A_{n}, A_{0}$, соответственно, что $\mu_{1} \subset \varepsilon\left(A_{1}\right), \ldots, \mu_{n} \subset \varepsilon\left(A_{n}\right), \varepsilon\left(A_{0}\right) \supset \mu_{0}$, то $\left(\mu_{1}, \ldots, \mu_{n}, \mu_{0}\right)$ - также инверсная конгруэнция на алгебре $\left(A_{1}, A_{2}, \ldots, A_{n}, A_{0} ; \omega\right)$. В этом случае будем говорить, что инверсная конгруэнция $\left(\mu_{1}, \ldots, \mu_{n}, \mu_{0}\right)$ является следствием инверсной конгруэнции $\varepsilon(\omega)$. На множестве инверсных конгруэнций на алгебре $\left(A_{1}, A_{2}, \ldots, A_{n}, A_{0} ; \omega\right)$ введем отношение $\leqslant$ следующего вида:

$$
\left(\mu\left(A_{1}\right), \ldots, \mu\left(A_{n}\right), \mu\left(A_{0}\right)\right) \leqslant\left(\varepsilon\left(A_{1}\right), \ldots, \varepsilon\left(A_{n}\right), \varepsilon\left(A_{0}\right)\right)
$$

если

$$
\mu\left(A_{1}\right) \subset \varepsilon\left(A_{1}\right), \ldots, \mu\left(A_{n}\right) \subset \varepsilon\left(A_{n}\right), \quad \varepsilon\left(A_{0}\right) \supset \mu\left(A_{0}\right) .
$$

Нетрудно заметить, что $\leqslant-$ отношение частичного порядка. Из определения отношения $\leqslant$ следует, что совокупность всех инверсных конгруэнций на алгебре $\left(A_{1}, A_{2}, \ldots, A_{n}, A_{0} ; \omega\right)$, не являющихся следствием друг друга, исчерпывается максимальными элементами относительно $\leqslant$. Такие инверсные конгруэнции будем называть максимальными.

\section{3. Конгруэнции координатных отношений}

Предложение 1. Если на алгебре $\left(A_{1}, \ldots, A_{n}, A_{0} ; \omega\right)$ существует конгруэнция $\left(\varepsilon(\omega), \omega^{*}\right)$, $\varepsilon(\omega)=\left(\varepsilon\left(A_{1}\right), \ldots, \varepsilon\left(A_{n}\right), \varepsilon\left(A_{0}\right)\right)$, координатного отношения $\rho$ такого, что не найдется элемента $\left[a_{0}\right] \varepsilon\left(A_{0}\right)$, для которого для любого $\left[a^{\prime}\right] \varepsilon\left(A_{0}\right) \in A_{0} / \varepsilon\left(A_{0}\right)$ справедливо включение $\left(\left[a^{\prime}\right] \varepsilon\left(A_{0}\right),\left[a_{0}\right] \varepsilon\left(A_{0}\right)\right) \in \rho$, то для каждого набора классов $\left[a_{1}\right] \varepsilon\left(A_{1}\right), \ldots,\left[a_{n}\right] \varepsilon\left(A_{n}\right)$ может быть так поставлен при соответствии $\omega^{* *}$ класс $\left[a_{0}\right] \varepsilon\left(A_{0}\right)$, что пара $\left(\varepsilon(\omega), \omega^{* *}\right)$ будет инверсной конгруэнчией на $\left(A_{1}, \ldots, A_{n}, A_{0} ; \omega\right)$.

Доказательство. По определению конгруэнции координатного отношения $\rho$ для любого набора $\left[a_{1}\right] \varepsilon\left(A_{1}\right), \ldots,\left[a_{n}\right] \varepsilon\left(A_{n}\right)$ найдется класс $\left[a_{0}\right] \varepsilon\left(A_{0}\right)$ такой, что

$$
\omega\left(\left[a_{1}\right] \varepsilon\left(A_{1}\right), \ldots,\left[a_{n}\right] \varepsilon\left(A_{n}\right)\right) \subset \bigcup_{b}[b] \varepsilon\left(A_{0}\right),
$$


где объединение берется по всем классам $[b] \varepsilon\left(A_{0}\right)$, находящимся с $\left[a_{0}\right] \varepsilon\left(A_{0}\right)$ в отношении $\rho$. По условию предложения, любому набору $\left[a_{1}\right] \varepsilon\left(A_{1}\right), \ldots,\left[a_{n}\right] \varepsilon\left(A_{n}\right)$ может быть в соответствии $\omega^{* *}$ поставлен такой класс $\left[a^{\prime}\right] \varepsilon\left(A_{0}\right)$, что

$$
\omega\left(\left[a_{1}\right] \varepsilon\left(A_{1}\right), \ldots,\left[a_{n}\right] \varepsilon\left(A_{n}\right)\right) \subset A_{0} \backslash\left[a^{\prime}\right] \varepsilon\left(A_{0}\right) .
$$

Таким образом, $\left(\varepsilon(\omega), \omega^{* *}\right)-$ инверсная конгруэнция алгебры $\left(A_{1}, \ldots, A_{n}, A_{0} ; \omega\right)$. Предложение доказано.

Конгруэнции координатного отношения $\rho$, для которых не выполняются условия предложения 1 , являются в некотором смысле тривиальными.

Предложение 2. Пусть $\rho$-естественное отношение линейного порядка $\leqslant$ на $E=\{0,1\}$ (то есть $0<1), f$ - булева функчия двух переменных такая, что для некоторых $\alpha_{1}, \alpha_{2} \in E$

$$
f\left(\alpha_{1}, \alpha_{2}\right)=f\left(\bar{\alpha}_{1}, \bar{\alpha}_{2}\right)=1,
$$

где $\bar{\alpha}$ - операчия инверсии.

Тогда любая квазигруппа $(A ; F)$, определенная на множестве $A,|A| \geqslant 2$, находится в гомоморфном координатном отношении $\leqslant c$ универсальной алгеброй $(E ; f)$.

Доказательство. Пусть

$$
F\left(a_{1}, a_{2}\right)=a_{3}
$$

определим отображения

$$
\varphi_{i}: A \rightarrow E, \quad i=0,1,2,
$$

следующим образом:

$$
\begin{aligned}
& \varphi_{1}\left(a_{1}\right)=\alpha_{1} \text { и } \varphi_{1}(a)=\bar{\alpha}_{1} \text { при } a \neq a_{1}, \\
& \varphi_{2}\left(a_{2}\right)=\alpha_{2} \text { и } \varphi_{2}(a)=\bar{\alpha}_{2} \text { при } a \neq a_{2}, \\
& \varphi_{0}\left(a_{3}\right)=1 \text { и } \varphi_{0}(a)=0 \text { при } a \neq a_{3} .
\end{aligned}
$$

Тогда для всех $a \neq a_{1}$ и $b \neq a_{2}$ справедливы соотношения

$$
\begin{aligned}
\varphi_{0}\left(F\left(a_{1}, a_{2}\right)\right) & =1, \\
\varphi_{0}\left(F\left(a_{1}, b\right)\right)=\varphi_{0}\left(F\left(a, a_{2}\right)\right) & =0, \\
f\left(\varphi_{1}\left(a_{1}\right), \varphi_{2}\left(a_{2}\right)\right)=f\left(\varphi_{1}(a), \varphi_{2}(b)\right) & =1,
\end{aligned}
$$

из которых следует справедливость отношения

$$
\varphi_{0}\left(F\left(a^{\prime}, b^{\prime}\right)\right) \leqslant f\left(\varphi_{1}\left(a^{\prime}\right), \varphi_{2}\left(b^{\prime}\right)\right)
$$

для всех $a^{\prime}, b^{\prime} \in A$.

Предложение доказано.

Для инверсных гомоморфизмов не существует утверждения, аналогичного предложению 2. А именно, существуют квазигруппы, которые не имеют в качестве инверсного гомоморфного образа универсальную алгебру $(E ; f)$, где $f-$ нетождественная булева 
функция. Чтобы показать это, заметим, что существование инверсного гомоморфизма алгебры $\left(A_{1}, \ldots, A_{n}, A_{0} ; \omega\right)$ на алгебру $\left(B_{1}, \ldots, B_{n}, B_{0} ; \vartheta\right)$, где $\left|B_{0}\right|=2$, влечет за собой существование гомоморфизма на эту же алгебру. Действительно, если $\left(\varphi_{1}, \ldots, \varphi_{n}, \varphi_{0}\right)$ - инверсный гомоморфизм, то для любого $\left(a_{1}, \ldots, a_{n}\right)$ из $A_{1} \times \ldots \times A_{n}$ справедливо соотношение

$$
\varphi_{0}\left(\omega\left(a_{1}, \ldots, a_{n}\right)\right) \neq \vartheta\left(\varphi_{1}\left(a_{1}\right), \ldots, \varphi_{n}\left(a_{n}\right)\right) .
$$

Определим отображение $\varphi_{0}^{\prime}$ множества $A_{0}$ на $B_{0}$, полагая

$$
\varphi_{0}^{\prime}(a)=B_{0} \backslash\left\{\varphi_{0}(a)\right\} .
$$

Так как $B_{0}$ состоит из двух элементов, определение корректно. Нетрудно видеть, что для любого $\left(a_{1}, \ldots, a_{n}\right) \in A_{1} \times \ldots \times A_{n}$ будет выполняться равенство

$$
\varphi_{0}^{\prime}\left(\omega\left(a_{1}, \ldots, a_{n}\right)\right)=\vartheta\left(\varphi_{1}\left(a_{1}\right), \ldots, \varphi_{n}\left(a_{n}\right)\right),
$$

то есть $\left(\varphi_{1}, \ldots, \varphi_{n}, \varphi_{0}^{\prime}\right)$ - гомоморфизм алгебры $\left(A_{1}, \ldots, A_{n}, A_{0} ; \omega\right)$ на алгебру $\left(B_{1}, \ldots, B_{n}, B_{0} ; \vartheta\right)$. Вместе с тем, любая абелева группа простого порядка не имеет нетривиальных гомоморфных образов (см. [7]) и, стало быть, не имеет в качестве инверсного гомоморфного образа универсальную алгебру $(E ; f)$.

Заметим также, что предложение 2 справедливо не для любой булевой функции $f$.

Предложение 3. Пусть $f$ - булева функция двух переменных, причем для некоторого $\alpha_{1} \in$ Е справедливы равенства

$$
f\left(\alpha_{1}, \alpha_{2}\right)=f\left(\alpha_{1}, \bar{\alpha}_{2}\right)=0 .
$$

Тогда для любой квазигруппь $(A ; F),|A| \geqslant 2$, не существует гомоморфного координатного отночения $\leqslant$, определенного в предложении 2 , с универсальной алгеброй $(E ; f)$.

Доказательство. Предположим противное. Пусть $\left(\varphi_{1}, \varphi_{2}, \varphi_{0}\right)$ - гомоморфизм координатного отношения $\leqslant$ квазигруппы $(A ; F)$ на $(E ; f)$. Тогда существуют элементы $a, b \in A$ такие, что $\varphi_{1}(a)=\alpha_{1}, \varphi_{0}(b)=1$. Так как $F-$ операция квазигруппы, найдется элемент $c \in A$ такой, что $F(a, c)=b$. Следовательно, $\varphi_{0}(F(a, c))=1, f\left(\varphi_{1}(a), \varphi_{2}(c)\right)=0$. Получаем противоречие, доказывающее утверждение.

Приведенные выше частные факты говорят о следующем: гомоморфизмы координатного отношения либо могут быть сведены к инверсным гомоморфизмам, либо в определенном смысле тривиальны.

Заметим, что на любой алгебре $\left(A_{1}, \ldots, A_{n}, A_{0} ; \omega\right),\left|A_{0}\right| \geqslant 2$, можно построить инверсную конгруэнцию вида $\left(\iota\left(A_{1}\right), \ldots, \iota\left(A_{n}\right), \varepsilon\left(A_{0}\right)\right)$, где $\iota(A)$ - отношение равенства на множестве $A$ (по крайней мере при $\left.\varepsilon\left(A_{0}\right)=\imath\left(A_{0}\right)\right)$. Поэтому будем говорить, что инверсная конгруэнция $\left(\varepsilon\left(A_{1}\right), \ldots, \varepsilon\left(A_{n}\right), \varepsilon\left(A_{0}\right)\right)$ нетривиальна, если среди отношений эквивалентности $\left(\varepsilon\left(A_{1}\right), \ldots, \varepsilon\left(A_{n}\right)\right.$ найдется отличное от отношения равенства.

Предложение 4. Для любой п-квазигруппы $(A ; F),|A|>2$, существует нетривиальная инверсная конгруэнщия.

Доказательство. Зададим на $A$ некоторое нетривиальное отношение эквивалентности $\varepsilon$, $i \in\{1, \ldots, n\}$. Тогда для набора эквивалентностей

$$
\varepsilon(F)=\left(\varepsilon_{1}(A), \ldots, \varepsilon_{n}(A), \varepsilon_{0}(A)\right),
$$


где

$$
\varepsilon_{1}(A)=\ldots=\varepsilon_{i-1}(A)=\varepsilon_{i+1}(A)=\ldots=\varepsilon_{n}(A)=\varepsilon_{0}(A)=\iota(A), \quad \varepsilon_{i}(A)=\varepsilon
$$

определяется инверсная конгруэнция $\left(\varepsilon(F), F^{*}\right)$ на $(A ; F)$, где $F^{*}$ ставит в соответствие каждому набору $\left(a_{1}, \ldots, a_{i-1},\left[a_{i}\right] \varepsilon, a_{i+1}, \ldots, a_{n}\right)$ элемент $a_{0}$ такой, что

$$
a_{0}=F\left(a_{1}, \ldots, a_{i-1}, a_{i}^{\prime}, a_{i+1}, \ldots, a_{n}\right), \quad a_{i}^{\prime} \notin\left[a_{i}\right] \varepsilon
$$

Предложение доказано.

Последнее утверждение говорит о новом качестве введенного понятия инверсного гомоморфизма, так как существуют $n$-квазигруппы, у которых отсутствуют нетривиальные гомоморфизмы (см. [7]). Данное утверждение также свидетельствует, что гомоморфные отношения не сводятся к гомоморфизмам алгебраических систем.

\section{4. О некоторых ограниченных гомоморфизмах n-квазигрупп}

Будем рассматривать конечные $n$-квазигруппы $(A ; F)$, построенные с использованием суперпозиций квазигрупп, изотопных некоторой одной квазигруппе $(A ; \cdot)$, следующего вида:

$$
F\left(x_{1} x_{2} \ldots x_{n}\right)=h_{n}\left(\ldots h_{3}\left(h_{2}\left(h_{1}\left(x_{1}\right) x_{2}\right) x_{3}\right) \ldots x_{n}\right)
$$

$x_{i} \in A, h_{i}$ - подстановка множества $A, i=1, \ldots, n$.

Данный класс $n$-квазигрупп исследовался в ряде работ ([8]-[11]) и представляет интерес как с теоретической, так и практической точек зрения. В частности, как показано в [8], подходящими суперпозициями таких $n$-квазигрупп можно получить любую $n$-квазигруппу порядка $k \geqslant 7$ (точные формулировки см. в [8]). Данный класс $n$-квазигрупп появляется и в ряде практических приложений, например, в криптографии. В частности, структуру такой $n$-квазигруппы задает преобразование блочного шифра, построенного по принципу SQUARE (например, алгоритм, принятый в качестве стандарта шифрования AES), а также ряд других известных блочных шифров, реализованных по принципу SP-сети.

Пусть в дальнейшем $(A ; \cdot)$ - абелева группа, $\varphi_{i}, i \in I,-$ ее гомоморфизмы такие, что $\left|\operatorname{Ker} \varphi_{i}\right|=k$. Мы будем рассматривать ограниченные гомоморфизмы определяемой (2) $n$-квазигруппы $(A ; F)$ вида

$$
\Phi=\left(\varphi_{i_{1}}, \ldots, \varphi_{i_{n}}, \varphi_{i_{n+1}}\right)
$$

Очевидно, что существуют $n$-квазигруппы $(A ; F)$, определяемые $(2)$, у которых существуют ограниченные гомоморфизмы (3) (например, те, у которых $Ф$ является гомоморфизмом). Поэтому вызывает интерес вопрос существования $n$-квазигрупп $(A ; F)$, у которых отсутствуют ограниченные гомоморфизмы (3).

Определим для каждой подстановки $h_{l}$ дважды стохастическую матрицу

$$
M_{l}=M_{l}\left(\operatorname{Ker} \varphi_{i_{l}}, \operatorname{Ker} \varphi_{i_{l+1}}\right)
$$

с элементами

$$
q_{t j}=k^{-1}\left|h_{l}\left(\left[a_{t}\right] \operatorname{Ker} \varphi_{i_{l}}\right) \cap\left[a_{j}\right] \operatorname{Ker} \varphi_{i_{l+1}}\right|
$$


Обозначим $T_{\alpha}\left(\varphi_{i}\right)(0,1)$-матрицу перестановок размера $\left|A / \operatorname{Ker} \varphi_{i}\right| \times\left|A / \operatorname{Ker} \varphi_{i}\right|$, элемент которой $q_{l j}=1$, если

$$
\left[a_{\alpha}\right] \operatorname{Ker} \varphi_{i} \times_{i}\left[a_{l}\right] \operatorname{Ker} \varphi_{i}=\left[a_{j}\right] \operatorname{Ker} \varphi_{i}
$$

$\alpha, l, j \in A / \operatorname{Ker} \varphi_{i}$ и $\times_{i}-$ операция в фактор-группе $A / \operatorname{Ker} \varphi_{i}$.

Для произведения матриц будем использовать обозначение

$$
U_{z}(F, \Phi)=M_{\mathrm{i}} \times T_{\alpha_{2}}\left(\varphi_{2}\right) \times \ldots \times M_{n-1} \times T_{\alpha_{n}}\left(\varphi_{n-1}\right) \times M_{n}
$$

где $z=\left(\alpha_{2}, \ldots, \alpha_{n}\right), \alpha_{i} \in A / \operatorname{Ker} \varphi_{i}, i=1, \ldots, n$.

Предложение 5. Пусть среди матрии $M_{l}, l=1, \ldots, n$, найдется $|A| / k-1$ вполне неразложимых. Тогда Ф не является ограниченным гомоморфизмом $(A ; F)$.

Доказательство. Матрицу $U_{z}(F, \Phi)$ можно рассматривать как матрицу переходных вероятностей за $n$ шагов простой неоднородной цепи Маркова, управляемой последовательностью матриц $M_{1} \times T_{\alpha_{2}}\left(\varphi_{2}\right), \ldots, M_{n-1} \times T_{\alpha_{n}}\left(\varphi_{n-1}\right), M_{n}$.

Полагая, что в начальный момент цепь Маркова находилась в некотором $i$-м состоянии, посмотрим во сколько $R_{l}$ состояний она может попасть через $l$ шагов, $l=1, \ldots, n, R_{0}=1$. Для получения оценки $R_{l}$ предварительно приведем вспомогательное утверждение.

Лемма 1. В любых $l$ строках, $l=1, \ldots, m-1$, дважды стохастической матричь $М$ размера $m \times m$ положительные элементы находятся в $t \geqslant l$ столбчах, если же $M$ вполне неразложима, то в $t>l$ столбиах.

Доказательство леммы 1. Напомним (см. [12]), что неотрицательная матрица $A$ называется частично разложимой, если существуют такие матрицы перестановок П и $\Pi_{1}$, что

$$
\Pi \times A \times \Pi_{1}=\left(\begin{array}{cc}
B & D \\
0 & C
\end{array}\right),
$$

где $B, C$ - квадратные матрицы. Если неотрицательная матрица не является частично разложимой, то она называется вполне неразложимой. Пусть $M$ - произвольная дважды стохастическая матрица и положительные элементы в строках с номерами $i_{1}, \ldots, i_{l}$ находятся в столбцах с номерами $j_{1}, \ldots, j_{t}$. Суммируя эти элементы, получаем соотношения

$$
\begin{aligned}
& \sum_{d=1}^{l} \sum_{f=1}^{t} P_{i_{d} j_{f}}=\sum_{d=1}^{l} 1, \\
& \sum_{f=1}^{t} \sum_{d=1}^{l} P_{i_{d} j_{f}} \leqslant t .
\end{aligned}
$$

Равенство дает частичную разложимость. Лемма доказана.

Согласно доказанной лемме, если матрица $M_{l+1}$ вполне неразложима, то $R_{l+\mathrm{i}} \geqslant R_{l}+1$, а если частично разложима, то $R_{l+1} \geqslant R_{l}$. Следовательно, через $n$ шагов цепь Маркова может оказаться в любом состоянии, или, что то же самое, матрица $U_{z}(F, \Phi)$ положительна, если вполне неразложимых среди матриц $M_{l}$ не меньше $r-1$. Положительность матрицы говорит об отсутствии ограниченного гомоморфизма. Предложение доказано.

Покажем, что существуют подстановки, удовлетворяющие условию предложения 5. 
Предложение 6. Пусть $\varepsilon_{i}, \mu_{i}, \varepsilon_{i+1}, \mu_{i+1},-$ конгруэнции на группе $(A ; \cdot)$ такие, что

$$
\left|A / \varepsilon_{i}\right|=\left|A / \varepsilon_{i+1}\right|, \quad\left|A / \mu_{i}\right|=\left|A / \mu_{i+1}\right|, \quad \mu_{i} \subset \varepsilon_{i}, \quad \mu_{i+1} \subset \varepsilon_{i+1} .
$$

Тогда, если матрича $M\left(\varepsilon_{i}, \varepsilon_{i+1}\right)$ частично разложима, то матрица $M\left(\mu_{i}, \mu_{i+1}\right)$ также частично разложима.

Доказательство. Если элемент

$$
q_{l j}=\left|\left[a_{l}\right] \varepsilon_{i}\right|^{-1}\left|h\left(\left[a_{l}\right] \varepsilon_{i}\right) \cap\left[a_{j}\right] \varepsilon_{i+1}\right|
$$

матрицы $M\left(\varepsilon_{i}, \varepsilon_{i+1}\right)$ равен нулю, то в матрице $M\left(\mu_{i}, \mu_{i+1}\right)$ равны нулю все элементы с индексами $(d, f)$ такими, что

$$
\left[a_{d}\right] \mu_{i} \subset\left[a_{l}\right] \varepsilon_{i}, \quad\left[a_{f}\right] \mu_{i+1} \subset\left[a_{j}\right] \varepsilon_{i+1} .
$$

Таким образом, если в матрице $M\left(\varepsilon_{i}, \varepsilon_{i+1}\right)$ сушествует собственная дважды стохастическая подматрица, стоящая на пересечении строк с номерами $p_{1}, \ldots, p_{s}$ и столбцов с номерами $q_{1}, \ldots, q_{s}$, то и в матрице $M\left(\mu_{i}, \mu_{i+1}\right)$ найдется собственная дважды стохастическая подматрица, элементы которой имеют индексы $(d, f)$ такие, что

$$
\left[a_{d}\right] \mu_{i} \subset\left[a_{p_{t}}\right] \varepsilon_{i}, \quad\left[a_{f}\right] \mu_{i+1} \subset\left[a_{q_{r}}\right] \varepsilon_{i+1}
$$

Предложение доказано.

Из предложения 6 следует, что для того, чтобы установить вполне неразложимость матрицы $M\left(\varepsilon_{i}, \varepsilon_{i+1}\right)$ достаточно показать вполне неразложимость матрицы $M\left(\mu_{i}, \mu_{i+1}\right)$.

Предложение 7. Пусть $\varepsilon$ и $\mu$-конгруэнции на группе $(A ; \cdot)$ такие, что

$$
|A / \varepsilon|=|A / \mu|=|A| / 2 \text {. }
$$

Тогда для любой подстановки $h$ матрича $M(\varepsilon, \mu)$ представима выпуклой комбиначией двух матрич перестановок $P_{1}, P_{2}$ вида

$$
M(\varepsilon, \mu)=\frac{1}{2} P_{1}+\frac{1}{2} P_{2}
$$

Доказательство. По теореме Биркгофа (см., например, [13]) любая дважды стохастическая матрица представима в виде выпуклой комбинации матриц перестановок. По определению матрицы $M(\varepsilon, \mu)$ любой ее элемент равен либо нулю, либо единице, либо 1/2. Следуя доказательству теоремы Биркгофа, выделим в матрице $M_{h}(\varepsilon, \mu)$ диагональ, все элементы которой положительны, $P_{1}$ - матрица перестановки с единичными элементами в позициях, соответствующих этой диагонали. Заметим, что

$$
M(\varepsilon, \mu)-\frac{1}{2} P_{1}=\frac{1}{2} P_{2}
$$

где $P_{2}$ - матрица перестановок, так как после вычитания в каждой строке и в каждом столбце получившейся матрицы должен стоять ненулевой элемент, равный $1 / 2$. Предложение доказано. 
Предложение 8. Если квадратная матрича $M$ размера $m \times m$ представима выпуклой комбиначией двух матрич перестановок $P_{1}, P_{2}$ вида

$$
M=\frac{1}{2} P_{1}+\frac{1}{2} P_{2}
$$

то она вполне неразложима тогда и только тогда, когда матрице перестановки $P_{1}^{-1} \times P_{2}$ соответствует подстановка полного чикла симметрической группы $S_{m}$.

Доказательство. Пусть выполняются условия теоремы и матрица $M$ частично разложима, то есть в ней существует собственная дважды стохастическая подматрица, находящаяся на пересечении строк с номерами $i_{1}, \ldots, i_{l}$ и столбцов с номерами $j_{1}, \ldots, j_{l}$, $1 \leqslant l<m$. Это означает, что подстановки $g_{1}, g_{2}$, соответствующие матрицам $P_{1}$ и $P_{2}$, переводят $i_{1}, \ldots, i_{l}$ в $j_{1}, \ldots, j_{l}$. Следовательно, в цикловом разложении подстановки $g^{-1} g_{2}$ найдутся циклы, полностью состоящие из $j_{1}, \ldots, j_{l}$.

Обратно, пусть в разложении подстановки $g^{-1} g_{2}$ на независимые циклы существуют циклы на $j_{1}, \ldots, j_{l}, 1 \leqslant l<m, g_{1}$ и $g_{2}$ переводят $i_{1}, \ldots, i_{l}$ в $j_{1}, \ldots, j_{l}$. Тогда в $M$ существует собственная дважды стохастическая подматрица, стоящая на пересечении строк с номерами $i_{1}, \ldots, i_{l}$ и столбцов с номерами $j_{1}, \ldots, j_{l}$. Следовательно, матрица $M$ частично разложима. Предложение доказано.

Доказанные предложения 6, 7,8 приводят к алгоритму построения подстановок, для которых матрицы $M\left(\operatorname{Ker} \varphi_{i_{l}}, \operatorname{Ker} \varphi_{i_{l+1}}\right)$ будут вполне неразложимы. Опишем этот алгоритм для аддитивной группы $V_{N}(+)$ кольца вычетов по модулю $2^{N}$. Любая нетривиальная конгруэнция $\sigma_{k}$ группы $V_{N}(+)$ имеет следующий вид: $(a, b) \in \sigma_{k}$ тогда и только тогда, когда

$$
a \equiv b \quad\left(\bmod 2^{k}\right), \quad k \in\{1, \ldots, N-1\} .
$$

Из предложения 6 следует, что для любого $1 \leqslant k<N$ матрица $M\left(\sigma_{k}, \sigma_{k}\right)$ вполне неразложима, если вполне неразложима матрица $M\left(\sigma_{N-1}, \sigma_{N-1}\right)$. Последняя же матрица будет согласно предложению 8 вполне неразложимой тогда и толькл тогда, когда матрице перестановок $P_{1}^{-1} \times P_{2}$ соответствует подстановка полного цикла. Построим подстановку $h$, удовлетворяющую этому условию. Выберем две подстановки $g_{1}, g_{2}$ симметрической группы подстановок множества $\left\{0,1, \ldots, 2^{N-1}-1\right\}$ так, чтобы произведение $g_{1}^{-1} g_{2}$ было полным циклом. Затем двумя произвольными способами выберем из каждого класса

$$
K_{i}=\left\{i, i+2^{N-1}\right\}, \quad i \in\left\{0,1, \ldots, 2^{N-1}-1\right\}
$$

по одному элементу и объединим их первый раз в множество $U_{1}$, второй раз в множество $W_{1},\left|U_{1}\right|=2^{N-1},\left|W_{1}\right|=2^{N-1}$. Введем обозначения

$$
U_{2}=V_{N} \backslash U_{1}, \quad W_{2}=V_{N} \backslash W_{1} .
$$

Определим $h$ следующим образом: если $a \in U_{1}$ и $a \in K_{i}$, то $h(a)=b$, где $b \in K_{g_{1}(i)}$, $b \in W_{1}$, если же $a \in U_{2}$ и $a \in K_{i}$, то $h(a)=b$, где $b \in K_{g_{2}(i)}, b \in W_{2}$.

Пусть в (2)

$$
h=h_{1}=\ldots=h_{n}, \quad(A ; \cdot)=V_{N}(+), \quad n>N / 2-1,
$$

тогда согласно предложению 5 у $n$-квазигруппы $(A ; F)$ отсутствуют ограниченные гомоморфизмы вида (3). 


\section{Список литературы}

1. Higgins P. J., Algebras with a scheme of operators. Math. Nachr. (1963) 27, 115-132.

2. Higgins P. J., Groupoids and categories. North-Holland, Amsterdam, 1973.

3. Горчинский Ю. Н., О л-гомоморфизмах конечных многоосновных универсальных алгебр. Дискретная математика (1999) 11, №2, 3-19.

4. Карпунин Г. А., Шапошников И. Г., Скрещенные гомоморфизмы конечных многоосновных универсальных алгебр с бинарными операциями. Дискретная математика (2000) 12, №2, 6684.

5. Кон П., Универсальная алгебра. Мир, Москва, 1968.

6. Мальцев А. И., Алгебраические системы. Наука, Москва, 1970.

7. Шапошников И. Г., О конгруэнциях конечных многоосновных универсальных алгебр. Дискретная математика (1999) 11, №3, 48-62.

8. Глухов М. М., Об $\alpha$-замкнутых классах и $\alpha$-полных системах функций $k$-значной логики. Дискретная математика. (1989) 1, №1, 16-21.

9. Глухов М. М., Задание конечных групп системами образующих. Труды по дискретной математике (1997) 1, 43-66.

10. Глухов М. М., О 2-транзитивных произведениях регулярных групп подстановок. Труды по дискретной математике (2000) 3, 37-52.

11. Шапошников И. Г., Метод использования $\delta$-сплетения универсальных алгебр для построения преобразований с заданными свойствами. Обозрение прикладной и промышленной математики (2002) 9, №3, 669-670.

12. Сачков В. Н., Введение в комбинаторные методы дискретной математики. Наука, Москва, 1982.

13. Маркус М., Минк Х., Обзор по теории матрич и матричных неравенств. Наука, Москва, 1972.

Статья поступила 16.03.2004. 